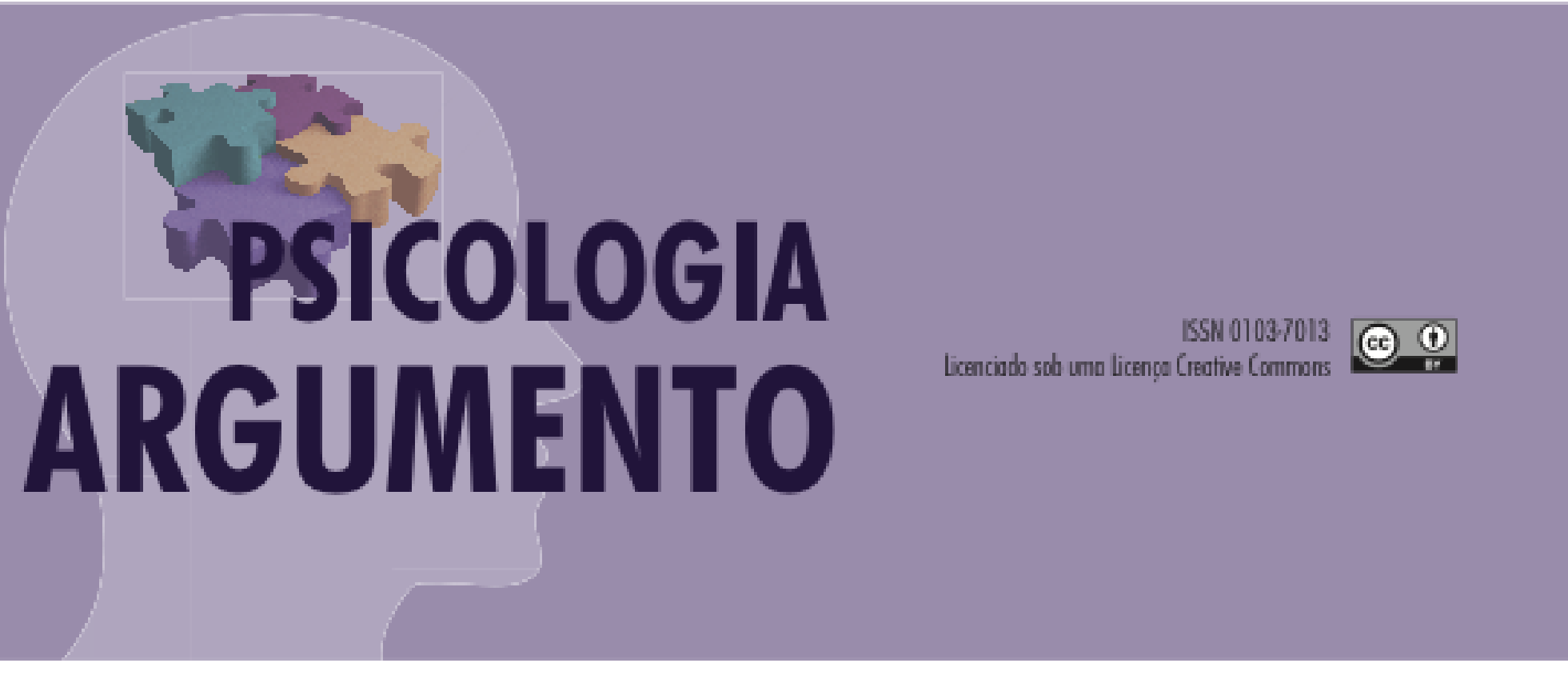

doi 10.7213/psicol.argum.33.080.AO04

\title{
Síndrome de Burnout em professores: estudo comparativo entre o ensino público e privado
}

\author{
Burnout syndrome in teachers: a comparative study between the \\ public and private education
}

Bruna Mainardi Rosso Borba ${ }^{[a]}$, Liciane Diehl ${ }^{[b]}$, Anelise Schaurich dos Santos ${ }^{[c]}$, Janine Kieling Monteiro[d], Angela Helena Marin ${ }^{[\mathrm{e}]}$

[a] Mestranda em Psicologia, Universidade do Vale do Rio dos Sinos (UNISINOS), São Leopoldo, Rio Grande do Sul, Brasil, bruna_borba@yahoo.com.br

[b] Doutoranda em Psicologia, Universidade do Vale do Rio dos Sinos (UNISINOS), São Leopoldo, Rio Grande do Sul, Brasil, licidiehl@gmail.com.

[c] Doutoranda em Psicologia, Universidade do Vale do Rio dos Sinos (UNISINOS), São Leopoldo, Rio Grande do Sul, Brasil, anelise_ssantos@hotmail.com

[d] Pesquisadora da linha de pesquisa Processos Saúde-Doença em Contextos Institucionais, Universidade do Vale do Rio dos Sinos (UNISINOS), São Leopoldo, Rio Grande do Sul, Brasil, janinekm@unisinos.br.

[e] Pesquisadora da linha de pesquisa Processos Saúde-Doença em Contextos Institucionais, Universidade do Vale do Rio dos Sinos (UNISINOS), São Leopoldo, Rio Grande do Sul, Brasil, marin.angelah@gmail.com

\section{Resumo}

Este estudo objetivou comparar a prevalência da Síndrome de Burnout entre professores de ensino fundamental de escolas públicas e privadas. Participaram 117 professores de ensino fundamental provenientes de escolas privadas $(54,7 \%)$ e públicas $(45,3 \%)$ das cidades de Porto Alegre e Região Metropolitana do Estado do Rio Grande do Sul/Brasil. Os participantes responderam a dois instrumentos autoaplicáveis: Questionário de Dados Sociodemográficos e Laboraise Cuestionario para laEvaluacióndel 
Síndrome de Quemarse por elTrabajo(CESQT). Realizaram-se análises estatísticas descritivas e o teste do qui-quadrado, que revelaram que a prevalência da síndrome é de $41,5 \%$ entre os professores das escolas públicas e $26,6 \%$ entre os da rede privada. Contudo, não houve diferença estatisticamente significativa entre os grupos $(\mathrm{p}=0,08)$. Concluiu-se que tanto o contexto público de ensino quanto o privado apresentam estressores que podem levar os professores ao adoecimento, dado que permite refletir que a prevalência da Síndrome de Burnout pode estar mais relacionada à categoria profissional em si do que ao setor de trabalho (público ou privado).

Palavras-chave: trabalho docente, saúde ocupacional, ensino público, ensino privado.

\begin{abstract}
The aim of this article was to compare the prevalence of burnout syndrome among primary school teachers from public and private schools. Participants were 117 primary school teachers from private $(54,7 \%)$ and public $(45,3 \%)$ schools of Porto Alegre and the Metropolitan Region of the State of Rio Grande do Sul/Brazil. Participants answered two self-report instruments: Socio-Demographic Data and Labor Questionnaire and Cuestionario para la Evaluacióndel Síndrome de Quemarse por el Trabajo (CESQT). Descriptive statistics analyses and chi-square test were performed. These revealed that the prevalence of the syndrome is $41.5 \%$ among public school teachers and $26.6 \%$ among private school teachers. However, there was no statistically significant difference between groups $(p=0.08)$. It was concluded that both labor contexts (public and private) present stressors that can lead teachers to illness, data that allows to reflect that the prevalence of Burnout Syndrome maybe is more related to professional category than the labor sector (public or private).
\end{abstract}

Keywords: teaching work, occupational health, public school education, private school education.

\title{
Introdução
}

A atividade docente tem sido alvo de muitas investigações, visto que no cotidiano de trabalho do professor estão presentes estressores psicossociais relacionados tanto à natureza de sua função, quanto ao contexto institucional e social em que estão inseridos (Lopes \& Pontes, 2009; Silveira, Enumo, \& Batista, 2014). A docência é considerada pela Organização Internacional do Trabalho (OIT) como uma atividade de risco desde 1981, uma vez que os professores compõem a segunda categoria profissional mais acometida por doenças ocupacionais em nível mundial (OIT, 2012).

Dentre as doenças ocupacionais em professores, as relacionadas a fatores psíquicos estão crescendo em muitos países (Guglielmi \& Tatrow, 1998). Como causas de adoecimento encontram-se: ritmo intenso e precárias condições de trabalho, aumento da exigência cognitiva, perda de autonomia em sala de aula, falta de acompanhamento técnico, políticas de educação insuficientes (Mendes, 2015), salários insatisfatórios, não reconhecimento social do trabalho (Monteiro, Dalagasperina, \& Quadros, 2012),indisciplina/violência e desinteresse dos alunos (Mesquita, Gomes, Lobato, Gondim, \& Souza, 2013; Monteiro et al., 2012). Nesse sentido, as condições e organização do trabalho do professor apresentam características que o expõem a fatores estressantes, os quais, se persistentes, podem levar à Síndrome de Burnout - SB (Guglielmi \& Tatrow, 1998).

A SB caracteriza-se como um fenômeno psicossocial que ocorre como uma resposta crônica aos estressores interpessoais ocorridos na situação de trabalho, 
principalmente quando existe excessiva pressão, conflitos, poucas recompensas emocionais e de reconhecimento (Gil-Monte, 2008a). Em virtude disso, a legislação brasileira, com a Lei $n^{\circ} 3.048 / 99$, contemplou a SB como doença do trabalho para as profissões que envolvem cuidados com saúde, educação e serviços humanos (Ministério da Saúde do Brasil, 1999).

A definição mais aceita e utilizada na literatura internacional sobre SB é a fundamentada na perspectiva psicossocial de Maslach e Jackson (1981), sendo esta constituída de três dimensões: (1) exaustão emocional, caracterizada pelo sentimento de carência em recursos emocionais e geralmente relacionado à sobrecarga de trabalho; (2) despersonalização, dimensão na qual o trabalhador desenvolve sentimentos negativos em relação às pessoas com as quais trabalha, acarretando em atitudes coerentes com estes sentimentos tais como indiferença e cinismo; e (3) baixa realização pessoal, identificada pela avaliação negativa no trabalho afetando o autoconceito, autoestima e relacionamentos pessoais do sujeito.

Mais recentemente, Gil-Monte (2005) desenvolveu um modelo de dimensões da SB fundamentado no já consolidado por Maslach e Jackson (1981), mas diferindo dele pela inclusão da dimensão culpa. As dimensões que compõem esse modelo são: (1) ilusão pelo trabalho, que indica o desejo individual para atingir metas relacionadas ao trabalho; (2) desgaste psíquico, relacionado ao sentimento de exaustão emocional e física devido ao contato direto com pessoas que são causadoras de problema; (3) indolência, referente à presença de atitudes de indiferença frente aos usuários do serviço prestado; e (4) culpa, que implica no sentimento gerado pelo comportamento e atitudes negativas desenvolvidas no trabalho (Gil-Monte, Carlotto, \& Câmara, 2010). Desse modelo resultam dois perfis. O perfil 1 é caracterizado pela baixa ilusão pelo trabalho e alto desgaste psíquico e indolência. O perfil 2 define a presença das dimensões descritas no Perfil 1, acrescidas da elevação do sentimento de culpa. Indivíduos classificados no último perfil tendem a apresentar maior absenteísmo (Gil-Monte, 2008b) e problemas de saúde (Carlotto, Librelotto, Pizzinato, \& Barcinski, 2012).

Nessa perspectiva, Costa, Gil-Monte, Possobona e Ambrosano (2013) investigaram a prevalência da $\mathrm{SB}$ em professores universitários da região Sudeste do país e os resultados revelaram que $11,2 \%$ dos professores apresentaram Perfil 1 e 3\%, Perfil 2. Esse resultado se assemelha ao encontrado no estudo de Gil-Monte, Carlotto e Câmara (2011) com professores de diferentes níveis de ensino da região Sul, pois se constatou que $12 \%$ dos participantes apresentavam altos níveis de SB (Perfil 1), e 5,6\% a sua forma mais grave (Perfil 2).

Segundo a Organização Mundial da Saúde (2008), em pesquisa realizada na Região Centro-Oeste do Brasil, a cada 100 professores da rede pública, 15 sofriam da SB, prevalecendo os seguintes sintomas: sentimento de baixa realização profissional, alto grau de esgotamento emocional e distanciamento dos alunos. Outro estudo envolvendo 265 professores de escolas públicas, realizado por Batista, Carlotto, Coutinho e Augusto (2010), indicou que 33,6\% dos docentes apresentaram alto nível de exaustão emocional, 43,4\% baixa realização profissional e 8,3\% altos índices de despersonalização, trazendo repercussões na saúde mental e no desempenho profissional dos docentes. Em contrapartida, a pesquisa de Mesquita et al. (2013), envolvendo 357 professores de escolas públicas, revelou que $15,74 \%$ dos professores apresentaram alto nível de exaustão emocional, sendo que apenas 3,31\% apresentou alto nível de despersonalização e nenhum 
professor relatou sentir baixa realização profissional, mesmo sobrecarregados com seu trabalho e com atitudes de indiferença entre colegas e alunos.

Um dos focos de estudos sobre SB tem sido abordar aspectos considerando as vivências e práticas dos docentes em instituições públicas e privadas, já que estes contextos se diferenciam. Em relação às escolas públicas, são apontados como fatores decisivos para o desenvolvimento da SB baixos salários, formação continuada deficitária para atender às demandas educacionais da atualidade, jornada excessiva de trabalho e insegurança frente à violência dos alunos (Levy, Sobrinho, \& Souza, 2009). Diehl e Carlotto (2014) observaram a indisciplina estudantil, a falta de apoio dos pais e da direção da escola, a sobrecarga de trabalho e a cobrança social como principais desencadeadores da SB. Já no ensino privado, Dalagasperina e Monteiro (2014) evidenciaram sentimento de insegurança diante de uma possível demissão correlacionado com a exaustão emocional. Tal instabilidade parece ser uma particularidade do ensino privado, uma vez que os professores da rede particular vivenciam mais frequentemente o medo de perder o emprego, especialmente nas situações de contenção de gastos e de crises financeiras.

Comparando os dois setores, Esteves-Ferreira, Santos e Rigolon (2014) identificaram que os professores da rede pública se mostraram mais propensos a desenvolver SB que os professores de instituições privadas. Os autores indicaram que os primeiros tendem a se afastar do trabalho mais frequentemente em função de problemas psicológicos e os segundos devido a problemas de saúde física. Já na pesquisa realizada por Vasconcelos, Granado e Marins Junior (2009) com 82 professores, a SB em docentes de escolas públicas resultou em $24,32 \%$, enquanto nas escolas privadas esse número foi de $13,33 \%$.

Nesse sentido, de acordo com a pesquisa realizada por Carlotto e Moraes (2010), que buscou verificar se existe diferença na relação entre variáveis demográficas e laborais e as dimensões da SB em uma amostra de 822 professores de escolas públicas e privadas, identificaram-se diferenças significativa nas três dimensões da SB. Os professores de escolas públicas apresentaram maior exaustão emocional e despersonalização, bem como menor realização no trabalho. Lopes e Pontes (2009) também constataram que professores de rede pública estadual possuem índices estatisticamente maiores em relação à dimensão exaustão emocional. No entanto, identificaram índices estatisticamente menores em relação à dimensão realização profissional comparados aos professores da rede particular de ensino.

Tais dados apontam para a necessidade de ampliação do conhecimento sobre as diferenças em relação a prevalência da SB em professores de escolas públicas e privadas, uma vez que estudos têm apresentado resultados distintos quando tais grupos são comparados. Ainda, a SB em professores mostra-se um problema social relevante, já que se encontra vinculada a grandes custos organizacionais ligados à rotatividade de pessoal, absenteísmo, problemas de produtividade e qualidade de ensino (Carlotto, 2012).

Frente ao exposto, este estudo objetivou comparar a prevalência da Síndrome de Burnout entre professores de ensino fundamental de escolas públicas e privadas. Acreditase que professores do ensino público apresentarão índices mais elevados da SB, já que os problemas no campo da educação, no Brasil, têm se mostrado mais sérios no tocante à instituição pública do que em relação à particular (Carlotto, 2012). Espera-se que os dados obtidos possam fundamentar o planejamento de medidas de prevenção e ações de 
intervenções mais eficazes voltadas a categoria docente, considerando as especificidades do contexto escolar.

\section{Método}

\section{Delineamento}

Trata-se de um estudo descritivo, comparativo e transversal, de abordagem quantitativa (Sampieri, Collado, \& Lucio, 2013).

\section{Participantes}

Participaram deste estudo 117 professores de ensino fundamental provenientes de escolas privadas $(54,7 \%)$ e públicas $(45,3 \%)$ das cidades de Porto Alegre e Região Metropolitana do Estado do Rio Grande do Sul/Brasil. Dentre os docentes da rede privada $(\mathrm{N}=64)$, a maioria $(\mathrm{n}=49)$ era do sexo feminino, assim como na rede pública $(\mathrm{N}=53)$, grande parte dos participantes também era do sexo feminino $(n=49)$. Quanto ao tempo médio de exercício da profissão, esse foi de 16,45 anos para os professores das escolas privadas $(\mathrm{DP}=9,91)$ e 15,71 anos para os docentes das escolas públicas $(\mathrm{DP}=6,61)$. Já o tempo médio de atuação em cada instituição foi de 10,22 anos $(\mathrm{DP}=8,56)$ para os participantes dos colégios privados e de 7,66 (DP $=6,76)$ para os participantes dos colégios públicos.

\section{Participantes}

Participaram deste estudo 117 professores de ensino fundamental provenientes de escolas privadas $(54,7 \%)$ e públicas $(45,3 \%)$ das cidades de Porto Alegre e Região Metropolitana do Estado do Rio Grande do Sul/Brasil. Dentre os docentes da rede privada $(\mathrm{N}=64)$, a maioria $(\mathrm{n}=49)$ era do sexo feminino, assim como na rede pública $(\mathrm{N}=53)$, grande parte dos participantes também era do sexo feminino $(n=49)$. Quanto ao tempo médio de exercício da profissão, esse foi de 16,45 anos para os professores das escolas privadas $(\mathrm{DP}=9,91)$ e 15,71 anos para os docentes das escolas públicas $(\mathrm{DP}=6,61)$. Já o tempo médio de atuação em cada instituição foi de 10,22 anos $(\mathrm{DP}=8,56)$ para os participantes dos colégios privados e de 7,66 (DP $=6,76)$ para os participantes dos colégios públicos.

\section{Instrumentos}

Os participantes responderam individualmente a dois instrumentos autoaplicáveis. O Questionário de Dados Sociodemográficos e Laborais, continha questões a respeito do sexo do professor, cidade onde atuava, tempo de exercício da profissão e tempo de trabalho na instituição. O Cuestionario para laEvaluacióndel Síndrome de Quemarse por El Trabajo (CESQT), validado para os docentes brasileiros por Gil-Monte, Carlotto e Câmara (2010), por sua vez, é composto 20 itens distribuídos em quatro subescalas: ilusão pelo trabalho (5 itens), desgaste psíquico (4 itens), indolência (6 itens) e culpa (5 itens). A amostra de validação do CESQT para o Brasil foi constituída por 714 professores que trabalhavam em instituições de ensino da cidade de Porto Alegre/Rio Grande do Sul e 
região metropolitana. $\mathrm{O}$ valor de alfa de Cronbach foi maior que 0,70 para todas as subescalas.

\section{Procedimentos de coleta e éticos}

Inicialmente, entrou-se em contato com a direção das escolas, a fim de pedir autorização para que a pesquisa fosse apresentada para os professores. Os diretores consentiram a realização da pesquisa por meio da assinatura do Termo de Concordância Institucional. Nas escolas onde o estudo pôde ser realizado, a divulgação e convite de participação aos professores foram feitos no intervalo dos períodos de aula. Nesse momento, os participantes foram informados sobre o caráter voluntário da pesquisa, o risco mínimo que ela apresentava, o sigilo em relação às informações coletadas e o anonimato da identidade dos participantes. Aqueles que concordaram em participar, assinavam o Termo de Consentimento Livre e Esclarecido (TCLE) em duas vias (uma para os participantes e uma para os pesquisadores) e, então, respondiam aos instrumentos da pesquisa. Destaca-se que o presente estudo foi submetido e aprovado pelo Comitê de Ética em Pesquisa da Universidade do Vale do Rio dos Sinos (Projeto N 12/084).

\section{Análise de dados}

Realizaram-se análises estatísticas descritivas para o levantamento dos dados do Questionário Sociodemográfico e Laboral. Também se executou o teste do qui-quadrado para comparar a prevalência da SB entre professores de ensino fundamental da rede pública e privada. Optou-se por esse teste porque os dados não apresentaram distribuição normal através do teste de Shapiro-Wilk. As análises foram conduzidas no programa StatisticalPackage for the Social Sciences (SPSS) for Windows (versão 20) e foi considerado estatisticamente o nível de significância de $p<0,05$.

A prevalência da SB foi definida a partir de altas pontuações em desgaste psíquico e indolência e baixa pontuação em ilusão pelo trabalho, compatível com o perfil 1 . Devido ao fato de ainda não existir dados de padronização da população brasileira, foi utilizada a classificação de percentis do instrumento original, baseada na população espanhola (GilMonte, 2008a).

\section{Resultados}

Os resultados são apresentados de maneira comparativa entre os grupos professores de escolas públicas e privadas. Tal comparação é relativa às médias obtidas nas dimensões da SB e a sua prevalência em cada um dos grupos. Observa-se, na Tabela 1, que os professores das escolas públicas apresentaram níveis mais baixos nas categorias ilusão pelo trabalho e mais altos na categoria indolência, apontando um risco maior para o desenvolvimento da $\mathrm{SB}$, apesar dessa diferença não ter sido estatisticamente significativa $(\mathrm{p}=0,08)$ entre os grupos. 
Tabela 1. Avaliação das Dimensões da Síndrome de Burnout

\begin{tabular}{lccc}
\hline & & Média & Desvio padrão \\
\cline { 3 - 4 } Ilusão pelo Trabalho & Pública & 2,8 & 0,7 \\
& Privada & 3,1 & 0,7 \\
Desgaste Psíquico & Pública & 2,0 & 1,0 \\
& Privada & 2,0 & 0,9 \\
Indolência & Pública & 1,1 & 0,5 \\
& Privada & 1,0 & 0,6 \\
Culpa & Pública & 0,9 & 0,6 \\
& Privada & 1,2 & 0,6 \\
\hline
\end{tabular}

Além disso, a prevalência de SB é proporcionalmente maior nos professores das escolas públicas. Contudo, não houve diferença estatisticamente significativa quanto à prevalência entre os grupos, conforme pode ser visto na Tabela 2.

Tabela 2. Comparação da Prevalência da Síndrome de

Burnout em Professores de Escolas Públicas e Privadas

\begin{tabular}{lcccc}
\hline & & Frequência & Porcentagem & $p$ \\
\cline { 3 - 5 } Escola pública & Não & 31 & 58,5 & \\
& Sim & 22 & 41,5 & \\
Escola privada & Não & 47 & 73,4 & 0,08 \\
& Sim & 17 & 26,6 & \\
\hline
\end{tabular}

\section{Discussão}

O objetivo deste estudo foi comparar a prevalência da SB entre professores do ensino fundamental de escolas públicas e privadas. Os resultados obtidos não confirmaram a hipótese levantada, visto que não houve diferença estatisticamente significativa entre os 
docentes de instituições públicas e privadas conforme a prevalência da SB $(x 2=2,91 ; \mathrm{gl}=1$; $\mathrm{p}=0,08)$. Apesar de os dados apontarem maior sintomatologia e prevalência da SB nos professores do âmbito público a diferença encontrada entre os grupos não foi expressiva.

Este resultado está de acordo com o estudo de Almeida, Silva, Centurion e Chiuz (2011), que buscou comparar níveis de SB entre professores de escola pública e privada e não identificou diferença significativa. Da mesma forma, a pesquisa de Bano e Malik (2014) não evidenciou diferença significativa no estresse ocupacional ao comparar professores paquistaneses de escolas de ambos os setores.

Pode-se entender esse resultado a partir de reflexões acerca das condições de trabalho dos professores, visto que pesquisas apontam que o exercício profissional da docência, tanto em instituições públicas quanto privadas, representam uma ocupação de elevado estresse (Lopes \& Pontes, 2009; Silveira, Enumo, \& Batista, 2014), afetando diretamente a saúde física e mental desses profissionais. Aguiar e Magajewski (2012) destacaram que docentes do ensino público e privado consideram a remuneração e a valorização desproporcional ao trabalho que executam para a sociedade, sendo, muitas vezes, o motivo de afastamentos ou doenças, já que se sentem insatisfeitos com a profissão e incomodados com a jornada desgastante de trabalho.

Por outro lado, Esteves-Ferreira et al.(2014) assinalaram que a insatisfação afeta os docentes de diferentes maneiras. Na rede pública, é desencadeada pelo desinteresse dos alunos, pela jornada de trabalho excessiva, bem como por baixos salários. Já na instituição privada, os docentes tendem a questionar o excesso de cobranças, mesmo em condições de adequada produtividade. No presente estudo, as médias obtidas na dimensão desgaste psíquico foram semelhantes em ambos os grupos, sugerindo que realmente o sentimento de exaustão emocional e física é relativo à profissão, independente do contexto.

Embora sejam conferidas aparentes vantagens no setor privado na medida em que os professores são considerados integrantes de um sistema de ensino que possibilita uma atuação de maior independência quanto aos seus recursos financeiros (Carlotto, 2012), a estrutura social vigente privilegia as leis do mercado e a escola passou a ser avaliada a partir de parâmetros de produtividade e eficiência empresarial. A chamada mercantilização do ensino vem acompanhada da exigência de resultados concretos, mensuráveis e imediatistas, principalmente porque os alunos e pais, pelo fato de estarem pagando exigem um diploma, como certificado de aprendizagem, o mais rápido possível. Ainda nessa perspectiva, a crescente expansão do ensino privado tem refletido o aumento do número de alunos atendendo ao paradigma da empresa-escola (mais clientes, maior lucratividade), bem como às expectativas do cliente financeiro: os pais dos alunos. Assim, para atender a essa demanda, o professor tem aumentado a sua carga horária laboral, resultando em maior esgotamento emocional (Dalagasperina \& Monteiro, 2014).

Quanto à realidade do ensino público, constata-se a necessidade de uma formação profissional de melhor qualidade para que o professor atenda as demandas a ele projetadas. Pereira e Allain (2006) sustentam que a expansão do sistema público de ensino e a consequente democratização do acesso à educação básica não foram seguidas por um correspondente investimento das verbas públicas destinadas à educação. Ainda, de um modo geral, os professores da escola pública estão em condições econômicas e sociais inferiores aos das escolas privadas, participam menos de atividades culturais e possuem menos acesso a meios tecnológicos. 
Portanto, embora as características de gestão e relação da escola pública e privada denotem culturas específicas, ambos os contextos contêm fatores que originam determinados estressores e desgaste emocional para o professor, podendo levar ao adoecimento. Professores tanto do ensino público como do privado estão suscetíveis ao sofrimento relacionado ao trabalho, ainda que, às vezes, por motivos diferentes (Carlotto, 2012). Apesar da diversidade dos aspectos contextuais e institucionais da realidade educacional brasileira, os sinais e sintomas apresentados pelos docentes repercutem em termos de saúde mental, podendo interferir no desempenho profissional com prejuízos importantes tanto na qualidade de seu trabalho quanto no processo de ensinoaprendizagem (Batista et al., 2010).

\section{Considerações finais}

Como visto, os dados obtidos nesta pesquisa não revelaram diferença significativa quanto a prevalência da SB entre professores do ensino fundamental de escolas públicas e privadas. Tal evidência possibilitou a reflexão de que essa síndrome pode estar mais relacionada à categoria profissional do que ao setor de atuação, público ou privado. Contudo, esse aspecto deve ser melhor investigado, uma vez que a literatura aponta divergências quanto às dimensões da SB em professores de instituições particulares e públicas (Esteves-Ferreira, et al., 2014; Vasconcelos, et al., 2009).

Destaca-se que os resultados apresentados dizem respeito a uma região específica, sem poder de generalização. Ademais, a amostra, mesmo sendo estatisticamente representativa, é pequena diante do número de 138.115 indivíduos que são professores da rede pública e privada no Rio Grande do Sul/Brasil, segundo dados fornecidos pela Secretaria da Educação do estado no ano de 2014. Por isso, sugere-se a realização de mais pesquisas sobre o tema, em diferentes regiões do Brasil e com mais participantes, visando obter um conjunto de conhecimento hábil para interpretação dos escores da SB padronizados à nossa realidade.

Também se identificou poucos estudos sobre Burnout comparando escolas públicas e privadas, preponderando aqueles com o foco no público-alvo envolvendo professores de escolas públicas. Esse fato pode estar relacionado ao acesso mais facilitado de pesquisadores neste contexto.

Em termos de intervenção, pode-se pensar em estratégias adequadas para abordar o estresse laboral, como trabalho preventivo ao surgimento da síndrome. Entende-se que os investimentos na saúde tornaram-se essenciais para as políticas de crescimento econômico em âmbito nacional e internacional, ainda mais quando se diz respeito à saúde do professor. Tanto quanto a saúde, a educação é condição preponderante para o desenvolvimento humano e social, pois possibilita aos indivíduos alcançarem a plenitude de suas potencialidades ao longo da vida. Neste sentido, é importante dar atenção às políticas públicas que estimulem e valorizem a profissão docente, permitindo que os professores manifestem sua dedicação e competência, mas também se sintam reconhecidos e estimulados profissionalmente. 


\section{Referências}

Lopes, A. P., \& Pontes, E. A. S. (2009). Síndrome de Burnout: Um estudo comparativo entre professores das redes pública estadual e particular. Psicologia Escolar e Educacional, 13(2), 275-281.Barreto, M. S. (2000). Uma jornada de humilhações. Dissertação de Mestrado em Psicologia Social, Pontifícia Universidade Católica de São Paulo, São Paulo.

Silveira, K. A., Enumo, S. R. F. \& Batista, E. P. (2014). Indicadores de estresse e estratégias de enfrentamento em professores de ensino multisseriado. Revista Quadrimestral da Associação Brasileira de Psicologia Escolar e Educacional, 18(3), 457-465.doi: $10.1590 / 2175-3539 / 2014 / 0183767$

Organização Internacional do Trabalho - OIT (2012). Cartilha sobre o trabalhador(a). Conceitos, direitos, deveres e informações sobre a relação de trabalho.

Guglielmi, R.S., \&Tatrow, K. (1998). Occupational stress, burnout, and health in teachers: a methodological and theoretical analysis. ReviewofEducationalResearch, 68(1), 61-69.

Mendes, M. L. M. (2015). A precarização do trabalho docente e seus efeitos na saúde dos professores da Rede Municipal de Ensino do Recife. Revista Humanae,9(1), 62-75. Bicalho, R. A. (2009). Das histórias de violência em uma empresa júnior à reprodução da ideologia da administração. Dissertação de mestrado, Universidade Federal de Minas Gerais, Belo Horizonte.

Monteiro, J. K., Dalagasperina, P., \& Quadros, M. O. (2012). Professores no limite: o estresse no trabalho do ensino privado do Rio Grande do Sul. Porto Alegre: Carta Editora.

Mesquita, A. A., Gomes, D. S., Lobato, J. L., Gondim, L., \& Souza, S. B. (2013). Estresse e Síndrome de Burnout em professores: prevalência e causas. Psicologia Argumento, 31(75), 627-635. doi: 10.7213/psicol.argum.31.075.DS05

Gil-Monte, P.R. (2008a). El síndrome de quemarse por eltrabajo (burnout) como fenómeno transcultural. Información Psicológica, 91(92), 4-11.

Brasil (1999). Decreto no 3.048, de 6 de maio de 1999. Aprova o Regulamento da Previdência Social, e dá outras providências. Retirado de http://www.planalto.gov.br/ ccivil_03/decreto/d3048compilado.htm

Maslach, C., \& Jackson (1981). The measurement of experienced burnout. JournalofOcuppationalBehavior, 2, 99-113.

Gil-Monte, P. R. (2005). El sindrome de quemarse por el trabajo ("burnout"): Una enfermedad laboral en la sociedad del bienestar. Madrid: Piramide.

Gil-Monte, P. R, Carlotto, M. S., \& Câmara, S. G. (2010). Validação da versão brasileira do "Cuestionario para La Evaluación del Síndrome de Quemarse por el Trabajo" emprofessores. Revista de SaúdePública, 44(1), 140-147. 
Gil-Monte, P. R. (2008b). Magnitude of relationship between burnout and absenteeism: A preliminary study. Psychological Reports, 102, 465-568.

Carlotto, M. S., Librelotto, R., Pizzinato, A., \&Barcinski, M. (2012). Prevalência e factores associados à Síndrome de Burnout nos professores de ensino especial. Análise Psicológica, 30(3), 315-327.

Costa, L. D. S. T., Gil-Monte, P. R., Possobon, R. F., \&Ambrosano, G. M. B. (2013). Prevalência da Síndrome de Burnout em uma amostra de professores universitários brasileiros. Psicologia: Reflexão e Crítica, 26(4), 636-642.

Gil-Monte, P. R., Carlotto, M. S., \& Câmara, S. (2011). Prevalence of burnout in a sample of Brazilian teachers. The EuropeanJournalofPsychiatry, 25, 205-212.

Organização Mundial da Saúde - OMS (2008). Relatório Mundial de Saúde. Cuidados de Saúde Primários - Agora mais do que nunca. Genebra.

Batista, J. B. V., Carlotto, M. S., Coutinho, A. S., \& Augusto, L. G. S. (2010). Prevalência da Síndrome de Burnout e fatores sociodemográficos e laborais em professores de escolas municipais da cidade de João Pessoa, PB. Revista Brasileira de Epidemiologia,13(3), 502-12. doi:10.1590/S1415-790X2010000300013.

Levy, G. C. T., Nunes, F. P., \& Souza, C. A. A. (2009). Síndrome de Burnout em professores da rede pública. ProductionJournal, 19(3), p. 458-465.doi:10.1590/S010365132009000300004 .

Diehl, L., \& Carlotto, M. S. (2014). Conhecimento de professores sobre a Síndrome de Burnout: Processo, fatores de risco e consequências. Psicologia em estudo, 19(4) 741752. doi: 10.1590/1413-73722455415.

Dalagasperina, P., \& Monteiro, J. K. (2014). Preditores da síndrome de burnout em docentes do ensino privado. Psico-USF, 19(2), 263-275.doi: 10.1590/141382712014019002011.

Esteves-Ferreira, A. A., Santos, D. E., \&Rigolon, R. G. (2014). Avaliação comparativa dos sintomas da Síndrome de Burnout em professores de escolas públicas e privadas. Revista Brasileira de Educação, 19(59), 987-1002.

Vasconcelos, F. F., Granado, I. E., \& Junior, J. M. (2009). Estudo comparativo sobre a incidência da Síndrome de Burnout em professores da rede pública e privada de Maringá-PR. Saúde e Pesquisa, 2(1), 23-26.

Carlotto, M. S.\& Moraes, M. G. (2010).Síndrome de Burnout e fatores associados em professores de escolas públicas e privadas. Boletim Academia Paulista de Psicologia, 30(79), 329-342.

Carlotto, M. S. (2012). Síndrome de Burnout em professores: avaliação, fatores associados e intervenção. Porto, Portugal: LivPsic.

Sampieri, R.H., Collado, C.F., \& Lucio. M. P. B. (2013). Metodologia de pesquisa. 
Porto Alegre: Penso.Dejours, C. A. (1991). Loucura do trabalho. Estudo de psicopatologia do trabalho. 4. ed. São Paulo: Cortez - Oboré.

Almeida, C. V., Silva, C., Centurion, P., \&Chiuzi, R. M. (2011). Síndrome de Burnout em professores: um estudo comparativo na região do Grande $\mathrm{ABC}$ paulista. Revista Eletrônica Gestão e Serviços, 2(1), 276-291.doi: 10.15603/21777284/regs.v2n1p276-291

Bano, S., \&Malik, S. (2014). Effect of Occupational Stress on Life Satisfaction among Private and Public School Teachers. Journal of Independent Studies and Research, 12(1), 17-27.

Aguiar, C. F., \&Magajewski, F. R. L. (2012). Condições de trabalho e saúde dos professores do ensino fundamental e médio de escolas públicas e privadas de Laguna/SC. Cadernos Acadêmicos, 4(2), 212-214.

Pereira, J. E. P., \& Allain R. L. (2006). Considerações acerca do professor-pesquisador: a que pesquisa e a que professor se refere essa proposta de formação? Olhar de professor, 9(2), 269-282.Freitas, M. E. (1999). Cultura organizacional: Identidade, sedução e carisma. Rio de Janeiro: FGV.

Recebido/Received: 30/10/2015

Aprovado / Approved: 22/12/2015 